\title{
Author Correction: Critical observations on the French Conseil d'État ruling on plant mutagenesis
}

Dennis Eriksson (1D) and Tomasz Zimny (D)

Correction to: Nature Plants https://doi.org/10.1038/s41477-020-00819-4, published online 9 December 2020.

In the version of this Correspondence originally published, ref. 4 was incorrect; it should have read 'Avis du HCB sur le projet de décret modifiant l'article D.531-2 du code de l'environnement (Haut Conseil des Biotechnologies, 2020); http://www.hautconseildesbiotechnologies. $\mathrm{fr} /$ fr/avis/avis-hcb-sur-projet-decret-modifiant-larticle-d531-2-code-lenvironnement?. This error has been corrected.

Published online: 11 January 2021

https://doi.org/10.1038/s41477-021-00847-8

(C) The Author(s), under exclusive licence to Springer Nature Limited 2021 